\title{
Chimie des processus biologiques
}

\section{Marc Fontecave}

\section{(2) OpenEdition}

Journals

Édition électronique

URL : https://journals.openedition.org/annuaire-cdf/15528

DOI : $10.4000 /$ annuaire-cdf. 15528

ISBN : 978-2-7226-0572-5

ISSN : 2109-9227

Éditeur

Collège de France

Édition imprimée

Date de publication : 30 décembre 2020

Pagination : 101-108

ISBN : 978-2-7226-0516-9

ISSN : 0069-5580

Référence électronique

Marc Fontecave, "Chimie des processus biologiques », L'annuaire du Collège de France [En ligne], 118| 2020, mis en ligne le 01 avril 2021, consulté le 22 août 2022. URL : http://journals.openedition.org/ annuaire-cdf/15528 ; DOI : https://doi.org/10.4000/annuaire-cdf.15528 


\title{
CHIMIE DES PROCESSUS BIOLOGIQUES
}

\author{
Marc FONTECAVE \\ Membre de l'Institut (Académie des sciences), \\ professeur au Collège de France
}

Mots-clés : chimie, processus biologiques, biocatalyse, métalloenzymes, catalyseurs bioinspirés

La série de cours "Catalyse hétérogène et activation de petites molécules (I) 》 est disponible, en audio et/ou en vidéo, sur le site internet du Collège de France (https:// www.college-de-france.fr/site/marc-fontecave/course-2017-2018.htm).

\section{ENSEIGNEMENT}

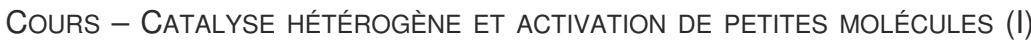

\section{Introduction}

L'eau, l'oxygène, l'azote, le dioxyde de carbone, etc., sont de petites molécules très abondantes à la surface de la planète et peuvent constituer des sources d'atomes presque infinies pour l'industrie chimique et nos sociétés. D'ailleurs ce sont elles que les organismes vivants utilisent depuis des milliards d'années pour leurs biosynthèses et métabolismes. Cependant, leur valorisation constitue un véritable défi scientifique et technologique en raison de leur très grande stabilité qui explique leur accumulation sur la terre et dans l'atmosphère. Pour les activer il faut des catalyseurs (dans les cellules vivantes il s'agit d'enzymes). Le cours porte spécifiquement sur la chimie de ces molécules et sur leur activation par une classe spécifique de catalyseurs : les catalyseurs hétérogènes, solides, qui sont probablement ceux qui sont considérés comme les plus prometteurs du point de vue des applications potentielles, du fait de leur plus grande stabilité et de leur recyclabilité. Cependant, un angle particulier est pris pour faire le lien entre cette classe de catalyseurs et les catalyseurs moléculaires homogènes (dont les enzymes naturelles font partie), à travers la description des approches d'hétérogénéisation des catalyseurs moléculaires, par exemple, d'utilisation de précurseurs moléculaires pour la synthèse de solides ou des approches de chimie hétérogène bioinspirée. 


\section{Cours 1 et 2 - Activation de petites molécules, des origines de la vie aux catalyseurs d'aujourd'hui}

Le premier cours s'attache à définir les différentes classes de catalyseurs (moléculaires, enzymatiques et solides), à comparer leurs avantages et leurs inconvénients respectifs et à montrer leur utilisation actuelle dans l'industrie. Il décrit également les réactions d'activation de petites molécules, $\mathrm{H}_{2} \mathrm{O}, \mathrm{H}_{2}, \mathrm{CO}_{2}, \mathrm{~N}_{2}, \mathrm{O}_{2}$, etc. Il montre comment ces molécules sont à l'origine chimique de la vie et décrit les sites actifs des métalloenzymes actuelles qui activent ces molécules et qui constituent pour le chimiste une source unique d'inspiration. Enfin, toujours sous forme d'introduction sur les catalyseurs hétérogènes, il montre comment aujourd'hui des techniques d'analyse d'une puissance inégalée permettent d'avoir des informations d'une très grand précision sur la structure de la surface des catalyseurs solides, y compris à l'échelle atomique, et comment les outils de la chimie théorique permettent de prévoir la réactivité des surfaces des solides sur lesquelles sont activées les petites molécules et de comprendre le mécanisme de ces réactions d'activation et de transformation.

\section{Cours 3 et 4 - Catalyse moléculaire : homogène ou hétérogène ?}

Les catalyseurs moléculaires, solubles, ont l'inconvénient d'une certaine instabilité, notamment dans les conditions parfois extrêmes que nécessite l'activation des petites molécules. Cette instabilité peut se traduire par une libération des ions métalliques et leur transformation en nanoparticules ou colloïdes métalliques. Même si ces composés peuvent se former en très faibles quantités, ils peuvent être en réalité des entités catalytiques puissantes, de sorte que la réaction observée n'est pas due au catalyseur moléculaire initial mais aux composés solides qui en dérivent. Ceci a conduit à de nombreuses erreurs d'interprétation dans la littérature et à de nombreuses controverses. Le cours s'appuie de façon détaillée sur des exemples très représentatifs de cette problématique qui montrent que certains catalyseurs moléculaires ne sont en fait que des « précatalyseurs ». Néanmoins, ces observations ouvrent de nouvelles perspectives en matière de synthèse de catalyseurs hétérogènes à partir de complexes organométalliques. En effet, le choix du précatalyseur, de son métal et de son ligand qui peut par synthèse être facilement modifié, a un impact considérable sur la structure et la réactivité des solides qui en dérivent. Ce concept est développé dans le cours.

\section{Cours 5 et 6 - Catalyseurs homogènes hétérogénéisés : matériaux à charpentes hybrides}

Une façon de synthétiser des catalyseurs solides de façon rationnelle et en gardant l'un des avantages de la catalyse homogène, à savoir la possibilité de modifier et de contrôler les activités du catalyseur par des modifications maîtrisées des coordinations du métal, est d'hétérogénéiser des catalyseurs moléculaires, c'est-àdire de fixer ces derniers sur une surface solide. Il y a de nombreuses façons de réaliser ces transformations de l'homogène à l'hétérogène. Le cours s'attache à traiter en profondeur une classe de supports solides d'un très grand intérêt: les polymères de coordination ou metal-organic frameworks (MOF). Ces solides poreux sont issus de la combinaison de centres métalliques (clusters le plus souvent) et de charpentes organiques. Ces dernières permettent aisément la fixation covalente de 
fonctions organiques et organométalliques, notamment des catalyseurs moléculaires. Les différentes stratégies de fixation sont présentées à partir de nombreux exemples significatifs. En général, après fixation les catalyseurs sont stabilisés, gardent leurs propriétés catalytiques et acquièrent donc certaines propriétés des catalyseurs solides, notamment leur recyclabilité. Enfin, les pores du matériau solide eux-mêmes peuvent constituer des sites de fixation de molécules. Cette fixation non covalente a été développée récemment, y compris dans notre laboratoire pour conduire à des catalyseurs homogènes hétérogénéisés originaux.

\section{COLLOQUE - PHOtOSYNTHÈSE ARTIFICIELLE ET CARBURANTS SOLAIRES}

Le colloque avait pour but de faire le point sur les recherches fondamentales et les technologies concernant la photosynthèse artificielle. Il s'agit ici de construire des dispositifs qui comportent les éléments suivants pour stocker, à la manière des organismes photosynthétiques, l'énergie solaire sous forme d'énergie chimique (carburants carbonés ou hydrogène): (i) des matériaux (semiconducteurs) ou molécules (photosensibilisateurs) pour la capture des photons solaires et pour séparer des charges; (ii) des catalyseurs utilisant les électrons excités pour la réduction de l'eau ou du $\mathrm{CO}_{2}$ et d'autres utilisant les trous pour oxyder l'eau en oxygène. Le colloque a traité de l'ensemble de ces éléments et de leur association au sein de cellules électrochimiques ou photoélectrochimiques.

\section{COURS À L'EXTÉRIEUR - CHIMIE ET DÉFIS ÉNERGÉTIQUES}

Trois sujets ont été traités sous la forme de trois cours :

(i) Chimie et défis énergétiques du $\mathrm{XXI}^{\mathrm{e}}$ siècle : une introduction.

(ii) Photosynthèse naturelle et photosynthèse artificielle.

(iii) Que faire du $\mathrm{CO}_{2}$ ? : de la chimie !

\section{RECHERCHE}

Le laboratoire de Chimie des processus biologiques développe des recherches à l'interface de la chimie et la biologie. Plus spécifiquement, il étudie des systèmes enzymatiques complexes impliqués dans des voies métaboliques et biosynthétiques, comme la modification des ARNs ou la biosynthèse de l'ubiquinone, du point de vue structural et mécanistique. Il s'intéresse également à certaines métalloenzymes du métabolisme bioénergétique comme les hydrogénases, qui catalysent la réduction de l'eau en hydrogène avec une remarquable efficacité et qui sont considérés comme des biocatalyseurs potentiels pour une utilisation dans des dispositifs électrochimiques de stockage d'énergie (bioélectrodes pour électrolyseurs et piles à combustibles). Enfin, les questions de la catalyse pour le stockage d'énergie sont traitées par des approches multiples en chimie de synthèse et en électrochimie, qui combinent de la chimie moléculaire bioinspirée et de la chimie du solide. Ces catalyseurs sont mis au point aussi bien pour l'oxydation de l'eau (catalyseurs pour l'anode des électrolyseurs), la réduction des protons en hydrogène et du dioxyde de carbone en composés organiques d'intérêt économique (catalyseurs pour la cathode des électrolyseurs).

Quelques faits marquants pour l'année académique 2017-2018 sur les quatre grands thèmes de recherche développés par le laboratoire sont résumés ci-dessous. 


\section{CATALYSE ET STOCKAGE D'ÉNERGIE}

Toute une série de nouveaux complexes moléculaires originaux, le plus souvent bioinspirés dans le sens où leur structure rappelle celle de certains sites actifs d'enzymes, à base de métaux non nobles comme le cobalt, le nickel ou le molybdène, ont été synthétisés et étudiés pour leurs propriétés catalytiques [C1, C3, C4, C7, C9, C10, C12, C17]. Certains d'entre eux ont révélé des efficacités catalytiques intéressantes aussi bien pour la réduction de protons en $\mathrm{H}_{2}$ ou la réduction du $\mathrm{CO}_{2}$ en monoxyde de carbone $\mathrm{CO}$. Leur réactivité est en général étudiée dans des conditions électrochimiques (l'énergie est d'origine électrique) mais dans certains cas aussi dans des conditions photochimiques (l'énergie est apportée par la lumière) [C6, C8, C14, C15, C18].

Le développement technologique de ces systèmes moléculaires nécessite que ces derniers soient hétérogénéisés. C'est ce qui a été récemment fait à travers la mise au point par le laboratoire de stratégies originales de fixation de certains de ces complexes moléculaires sur des solides poreux (metal-organic frameworks-MOFs) et des polymères de coordination [C14, C16, C18]. C. Mellot-Draznieks développe en propre divers sujets très fondamentaux sur les propriétés physico-chimiques des MOFs, par des approches computationnelles en particulier [C19-C21]. Le laboratoire met également au point des catalyseurs solides, nanostructurés et poreux, aussi bien pour la réduction des protons et du $\mathrm{CO}_{2}$ que pour l'oxydation de l'eau [C2, C5, C11, C13].

\section{HYDROGÉNASES}

Le laboratoire s'est engagé depuis quelques années dans un projet d'étude des hydrogénases à fer et de leur maturation. Ces enzymes sont d'excellents biocatalyseurs pour la production d'hydrogène. Leur structure complexe, la nécessité d'une étape de maturation pour la production d'une enzyme active et leur grande sensibilité à l'oxygène rendent leur utilisation dans des dispositifs technologiques cependant difficile. Nous étudions différentes stratégies pour résoudre ces questions : (i) une ingénierie rationnelle des hydrogénases par les outils de la biologie moléculaire; (ii) l'élaboration d'hydrogénases artificielles qui sont des systèmes hybrides associant une protéine hôte et un catalyseur de synthèse [H1, H2, H3].

\section{ENZYMES DE MODIFICATION DES ARNS}

La structure d'enzymes en complexe avec des substrats macromoléculaires (protéines, ADNs, ARNs, etc.) et la compréhension de leurs mécanismes de reconnaissance et d'action constituent encore aujourd'hui de formidables défis de la chimie biologique. Nous nous intéressons plus particulièrement aux enzymes de modification des ARNs de transfert qui jouent un rôle capital dans la traduction de l'information génétique. Plus particulièrement nous étudions deux classes d'enzymes : les protéines fer-soufre [M1, M3, M6] et les flavoprotéines [M4, M5].Tout récemment, par exemple, en combinant des approches structurales et biochimiques nous avons découvert un nouveau mécanisme de sulfuration des ARNs de transfert qui fait intervenir un centre fer-soufre pour le transfert des atomes de soufre [M2]. D. Hamdane, responsable du projet flavoprotéines pour la modification des ARNs de transfert en collaboration avec M. Lombard, a publié un important article de revue sur le domaine [M9] et travaille spécifiquement sur la dihydrouridine synthase [M8]. 


\section{ENZYMES DE LA BIOSYNTHĖSE DE L'UBIQUINONE}

L'ubiquinone est un cofacteur essentiel de la bioénergétique. Pourtant sa biosynthèse est encore mal connue en dépit du fait qu'elle constitue un véritable défi chimique en raison de la complexité de sa structure et de son insolubilité dans l'eau. Depuis plusieurs années, nous nous intéressons au complexe multiprotéique et multienzymatique impliqué dans la biosynthèse de l'ubiquinone. Tout récemment, nous avons découvert un nouvel acteur de ce processus biosynthétique, un assemblage de deux protéines qui semble jouer un rôle majeur dans la stabilisation du complexe biosynthétique et le transport de précurseurs lipidiques de l'ubiquinone [U1].

\section{Publications}

\section{Catalyse et stockage d'énergie [C1-C18]}

[C1] Roy S., Sharma B., Pecaut J., Simon P., Fontecave M., Tran P.D., Derat E. et ARTERO V., « Molecular cobalt complexes with pendant amines for selective electrocatalytic reduction of carbon dioxide to formic acid », Journal of the American Chemical Society, vol. 139, nº 10, 2017, p. 3685-3696, DOI : 10.1021/jacs.6b11474.

[C2] Huan T.N., Simon P., Rousse G., Genois I., Artero V. et Fontecave M., « Porous dendritic copper: An electrocatalyst for highly selective $\mathrm{CO}_{2}$ reduction to formate in water/ ionic liquid electrolyte », Chemical Science, vol. 8, no 1, 2017, p. 742-747, DOI : 10.1039/ c6sc03194c.

[C3] Elgrishi N., Chambers M.B., Wang X. et Fontecave M., « Molecular polypyridinebased metal complexes as catalysts for the reduction of $\mathrm{CO}_{2}$ », Chemical Society Reviews, vol. 46, no 3, 2017, p. 761-796, DOI : 10.1039/c5cs00391a.

[C4] Salomon W., Paille G., Gomez-Mingot M., Mialane P., Marrot J., RochMarchal C., Nocton G., Mellot-Draznieks C., Fontecave M. et DolbecQ A., « Effect of cations on the structure and electrocatalytic response of polyoxometalate-based coordination polymers », Crystal Growth \& Design, vol. 17, no 4, 2017, p. 1600-1609, DOI : 10.1021/acs. cgd.6b01600.

[C5] Huan T.N., Ranjbar N., Rousse G., Sougrati M., Zitolo A., Mougel V., Jaouen F. et FONTECAVE M., «Electrochemical reduction of $\mathrm{CO}_{2}$ catalyzed by Fe-N-C materials: A structure-selectivity study », ACS Catalysis, vol. 7, no 3, 2017, p. 1520-1525, DOI : 10.1021/ acscatal.6b03353.

[C6] Wang X., Goudy V., Genesio G., Maynadié J., Meyer D. et Fontecave M., «Ruthenium-cobalt dinuclear complexes as photocatalysts for $\mathrm{CO}_{2}$ reduction », Chemical Communications, vol. 53, no 36, 2017, p. 5040-5043, DOI : 10.1039/c6cc09941f.

[C7] Ching H.Y.V., Wang X., He M., Perujo Holland N., Guillot R., Slim C., Griveau S., Bertrand H.C., Policar C., Bedioui F. et Fontecave M., "Rhenium complexes based on 2-Pyridyl-1,2,3-triazole ligands: A new class of $\mathrm{CO}_{2}$ reduction catalysts », Inorganic Chemistry, vol. 56, $\mathrm{n}^{\circ}$ 5, 2017, p. 2966-2976, DOI : 10.1021/acs.inorgchem.6b03078.

[C8] Chambers M.B., Wang X., Ellezam L., Ersen O., Fontecave M., Sanchez C., Rozes L. et MELlot-DrazNIEKS C., «Maximizing the photocatalytic activity of metalorganic frameworks with aminated-functionalized linkers: Substoichiometric effects in MIL125-NH2 », Journal of the American Chemical Society, vol. 139, n 24, 2017, p. 8222-8228, DOI : $10.1021 /$ jacs.7b02186.

[C9] Aroua S., Todorova T.K., Hommes P., Chamoreau L.-M., Reissig H.-U., Mougel V. et FonteCAVE M., «Synthesis, characterization, and DFT analysis of bis-terpyridyl-based 
molecular cobalt complexes », Inorganic Chemistry, vol. 56, 2017, p. 5930-5940, DOI : 10.1021/acs.inorgchem.7b00595.

[C10] Fogeron T., Retailleau P., Chamoreau L.-M., Fontecave M. et Li Y., « The unusual ring scission of a quinoxaline-pyran-fused dithiolene system related to molybdopterin ", Dalton Transactions, vol. 46, $\mathrm{n}^{\circ}$ 13, 2017, p. 4161-4164, DOI : 10.1039/ C7DT00377C.

[C11] Huan T.N., Rousse G., Zanna S., LuCas I.T., Xu X., Menguy N., Mougel V. et FONTECAVE M., «A dendritic nanostructured copper oxide electrocatalyst for the oxygen evolution reaction », Angewandte Chemie International Edition, vol. 56, n 17, 2017, p. 47924796, DOI : 10.1002/anie.201700388.

[C12] Aroua S., Todorova T.K., Mougel V., Hommes P., Reissig H.-U. et FonteCave M., « New cobalt-bisterpyridyl catalysts for hydrogen evolution reaction», Chemcatchem, vol. 9, $\mathrm{n}^{\mathrm{o}}$ 12, 2017, p. 2099-2105, DOI : $10.1002 /$ cctc.201700428.

[C13] Soliman A.B., Hassan M.H., Huan T.N., Abugable A.A., Elmehalmey W.A., KaraKalos S.G., Tsotsalas M., Heinle M., Elbahri M., FonteCaVe M. et AlKordi M.H., «Pt immobilization within a tailored porous-organic polymer graphene composite: Opportunities in the hydrogen evolving reaction », Acs Catalysis, vol. 7, $\mathrm{n}^{\circ} 11,2017$, p. 7847 7854, DOI : 10.1021/acscatal.7b02246.

[C14] Wang X., Thiel I., Fedorov A., Coperet C., Mougel V. et Fontecave M., « Siteisolated manganese carbonyl on bipyridine-functionalities of periodic mesoporous organosilicas: efficient $\mathrm{CO}_{2}$ photoreduction and detection of key reaction intermediates », Chemical Science, vol. 8, $\mathrm{n}^{\circ}$ 12, 2017, p. 8204-8213, DOI : 10.1039/c7sc03512h.

[C15] Fontecave T. et FonteCAVE M., « Photosynthèse artificielle: transformer le soleil en carburants », Bulletin de l'Union des physiciens, vol. 1000, 2018, p. 249-260.

[C16] Paille G., Gomez-Mingot M., Roch-Marchal C., Lassalle-Kaiser B., Mialane P., Fontecave M., Mellot-Draznieks C. et DolbecQ A., « A fully noble metalfree photosystem based on cobalt-polyoxometalates immobilized in a porphyrinic metalorganic framework for water oxidation », Journal of the American Chemical Society, vol. 140, $\mathrm{n}^{\circ} 10,2018$, p. $3613-3618$, DOI : $10.1021 /$ jacs.7b11788.

[C17] Fogeron T., Todorova T.K., Porcher J.-P., GomeZ-Mingot M., Chamoreau L.-M., Mellot-DraznieKs C., Li Y. et FonteCaVe M., «A bioinspired nickel(bis-dithiolene) complex as a homogeneous catalyst for carbon dioxide electroreduction », ACS Catalysis, vol. 8, nº 3, 2018, p. 2030-2038, DOI : 10.1021/acscatal.7b03383.

[C18] Wang X., Wisser F.M., Canivet J., Fontecave M. et Mellot-Draznieks C., «Immobilization of a full photosystem in the large-pore MIL-101 metal-organic framework for $\mathrm{CO}_{2}$ reduction », ChemSusChem, vol. 11, no 18, 2018, p. 3315-3322, DOI : 10.1002/ cssc.201801066.

[C19] Mellot-Draznieks C. et CheEtham A.K., «Encoding evolution of porous solids », Nature Chemistry, vol. 9, no 1, 2017, p. 68, DOI : 10.1038/nchem.2700.

[C20] Bueken B., Van Velthoven N., Krajnc A., Smolders S., Taulelle F., MellotDraznieKs C., MALi G., BenNetT T.D. et DE Vos D., « Tackling the defect conundrum in UiO-66: A mixed-linker approach to engineering missing linker defects », Chemistry of Materials, vol. 29, 2017, p. 10478-10486.

[C21] Gomez G.E., Brusau E.V., Kaczmarek A.M., Mellot-Draznieks C., SaCanell J., Rousse G., Van Deun R., SAnchez C., NARDa G.E. et Soler Illia G.J.A.A., « Flexible ligand-based lanthanide three-dimensional metal-organic frameworks with tunable solid-state photoluminescence and $\mathrm{OH}$-solvent-sensing properties », European Journal of Inorganic Chemistry, $\mathrm{n}^{\mathrm{0}}$ 17, 2017, p. 2321-2331, DOI : 10.1002/ejic.201700099 


\section{Hydrogénases [H1-H3]}

[H1] Caserta G., Pecqueur L., Adamska-Venkatesh A., Papini C., Roy S., Artero V., AtTA M., ReIJERSE E., Lubitz W. et FonTECAVE M., « The [FeFe]-hydrogenase maturation protein HydF: Structural and functional characterization », Nature Chemical Biology, vol. 13, 2017, p. 779-784.

[H2] Caserta G., Papini C., Adamska-Venkatesh A., Pecqueur L., Sommer C., Reijerse E., Lubitz W., Gauquelin C., Meynial-Salles I., Pramanik D., Artero V., Atta M., Del Barrio M., Faivre B., Fourmond V., Léger C. et Fontecave M., «Engineering an [FeFe]-hydrogenase: Do accessory clusters influence $\mathrm{O}_{2}$ resistance and catalytic bias? », Journal of the American Chemical Society, vol. 140, $\mathrm{n}^{\circ} 16,2018$, p. 55165526, DOI : 10.1021/jacs.8b01689.

[H3] Sommer C., Rumpel S., Roy S., FArÈs C., Artero V., FonteCaVE M., ReiJerse E. et LUBITZ W., «Spectroscopic investigations of a semi-synthetic [FeFe] hydrogenase with propane di-selenol as bridging ligand in the binuclear subsite : comparison to the wild type and propane di-thiol variants », Journal of biological inorganic chemistry, vol. 23, $\mathrm{n}^{\mathrm{o}} 3,2018$, p. 481-491, DOI : 10.1007/s00775-018-1558-4.

\section{Métalloprotéines and flavoprotéines: modification d'ARNs [M1-M9]}

[M1] Altamura L., Horvath C., Rengaraj S., Rongier A., Elouarzaki K., Gondran C., Maçon A.L.B., Vendrely C., Bouchiat V., FontecaVe M., Mariolle D., Rannou P., Le GofF A., Duraffourg N., Holzinger M. et Forge V., «A synthetic redox biofilm made from metalloprotein-prion domain chimera nanowires », Nature Chemistry, vol. 9, n 2, 2017 , p. 157-163, DOI : 10.1038/nchem.2616.

[M2] ArRagain S., Bimai O., Legrand P., Caillat S., Ravanat J.-L., Touati N., Binet L., Atta M., Fontecave M. et Golinelli-Pimpaneau B., « Nonredox thiolation in tRNA occurring via sulfur activation by a $[4 \mathrm{Fe}-4 \mathrm{~S}]$ cluster $»$, Proceedings of the National Academy of Sciences of the United States of America, vol. 114, $\mathrm{n}^{\mathrm{o}} 28,2017$, p. 7355-7360, DOI: 10.1073/pnas.1700902114.

[M3] Mulliez E., Duarte V., Arragain S., Fontecave M. et AtTa M., «On the role of additional [4Fe-4S] clusters with a free coordination site in radical-SAM enzymes », Frontiers in Chemistry, vol. 5, 2017, DOI : 10.3389/fchem.2017.00017.

[M4] Bou-Nader C., Cornu D., Guerineau V., Fogeron T., Fontecave M. et HAMDANE D., «Enzyme activation with a synthetic catalytic coenzyme intermediate: Nucleotide methylation by new flavoenzymes », Angew. Chem. Int. Ed., vol. 56, 2017, p. 12523-12527.

[M5] Bou-Nader C., Pecqueur L., Cornu D., Lombard M., Dezi M., Nicaise M., VElours C., FonTECAVE M. et HAMDANE D., « Power of protein/tRNA functional assembly against aberrant aggregation », Physical Chemistry Chemical Physics, vol. 19, no 41, 2017, p. 28014-28027, DOI : 10.1039/c7cp05599d.

[M6] Py B., Gerez C., Huguenot A., Vidaud C., Fontecave M., Ollagnier de ChOudens S. et BARRAS F., «The ErpA/NfuA complex builds an oxidative resistant Fe-S cluster delivery pathway ", The Journal of Biological Chemistry, vol. 293, no 20, 2018, p. 7689-7702, DOI : 10.1074/jbc.RA118.002160.

[M7] Mazmouz R., Essadik I., Hamdane D., MÉJEAn A. et Ploux O., « Characterization of CyrI, the hydroxylase involved in the last step of cylindrospermopsin biosynthesis: Binding studies, site-directed mutagenesis and stereoselectivity », Arch. Biochem. Biophys., vol. 647, 2018, p. 1-9.

[M8] Bou-Nader C., Montémont H., Guérineau V., Jean-Jean O., Brégeon D. et HAMDANE D., «Unveiling structural and functional divergences of bacterial tRNA 
dihydrouridine synthases: Perspectives on the evolution scenario », Nucleic Acids Res., vol. 46, 2018, p. 1386-1394.

[M9] LOMBARD M. et HAMDANE D., « Flavin-dependent epitranscriptomic world », Archives of Biochemistry and Biophysics, vol. 632, 2017, p. 28-40, DOI : 10.1016/j.abb.2017.06.011.

\section{Biosynthèse de l'ubiquinone [U1]}

[U1] Loiseau L., Fyfe C., Aussel L., Haju Chehade M., Hernández S.B., Faivre B., Hamdane D., Mellot-Draznieks C., Rascalou B., Pelosi L., Velours C., Cornu D., Lombard M., Casadesús J., Pierrel F., FontecaVe M. et Barras F., « The UbiK protein is an accessory factor necessary for bacterial ubiquinone (UQ) biosynthesis and forms a complex with the UQ biogenesis factor UbiJ », The Journal of Biological Chemistry, vol. 292, $\mathrm{n}^{\mathrm{0}} 28$, 2017, p. 11937-11950, DOI : 10.1074/jbc.M117.789164. 